\title{
Contribution of glia cells specifically astrocytes in the pathology of depression: immunohistochemical study in different brain areas
}

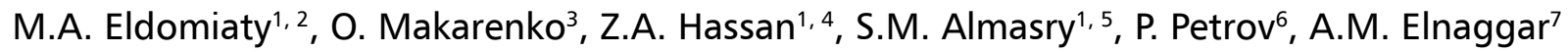 \\ ${ }^{1}$ Department of Anatomy, Faculty of Medicine, Taibah University, Saudi Arabia \\ 2Department of Anatomy, Faculty of Medicine, Tanta University, Egypt \\ IInternational Academy of Education Science, Bogomolets National Medical University, Kyiv, Ukraine \\ ${ }^{4}$ Department of Histology and Cell Biology, Faculty of Medicine, Zagazig University, Egypt \\ ${ }^{5}$ Department of Anatomy, Faculty of Medicine, Mansoura University, Egypt \\ ${ }^{6}$ Institute of Food Resources, Bogomolets National Medical University, Kyiv, Ukraine \\ ${ }^{7}$ Anatomy and Physiology Instructor, Academia Medical Institute, Columbus, Ohio, United States
}

[Received: 4 December 2019; Accepted: 14 January 2020]

Background: The contribution of glial cells to the pathophysiology of depression is an emerging research purpose. This study investigated the deficits in glial cells, specifically astrocytes in various brain regions, after the development of depression and then after voluntary running in depressed rats.

Materials and methods: Forty-five adult male Wistar rats aged 8-10 weeks were used in the study. A depression model was generated through a forced swimming programme; voluntary running was allowed on rat running wheels; and brain sections were taken from the hippocampus, dentate gyrus $(D G)$, medial prefrontal cortex (MPFC) and cerebellar cortex. After immunostaining with specific antibodies immuno-stain, the astrocytes, oligodendroglia and microglial cells were counted, and certain indices relating astrocytes to other glial cells were calculated. Astrocytic morphology was studied, and the optical density (OD) of glial fibrillary acidic protein (GFAP) immuno-expression was measured in different groups.

Results: Compared to the control group, animals in the depression group exhibited significant decreases in the mean astrocyte count in all studied brain areas, significant decreases in GFAP OD values in all areas and significantly reduced values for all glial astrocyte indices in the hippocampus, DG and mPFC. Compared to the rats in the depression group, those in the depression/exercise group exhibited significantly elevated mean astrocyte and oligodendroglia counts in all studied areas, significantly elevated GFAP OD values in all studied areas, and non-significant differences in glial astrocyte indices in the hippocampus, MPFC and cerebellar cortex.

Conclusion: Astrocytes, rather than other glia, constitute a basis for the development and/or relief of depression. (Folia Morphol 2020; 79, 3: 419-428)

Key words: stress, dentate gyrus, hippocampus, cerebellum, frontal cortex, glial cells, voluntary running

Address for correspondence: Dr. M.E. Eldomiaty, Department of Anatomy, Faculty of Medicine, Taibah University, Saudi Arabia; Department of Anatomy, Faculty of Medicine, Tanta University, Egypt, Postal code 30001, tel. 00966559579980, e-mail: dr_majda@hotmail.com 


\section{INTRODUCTION}

Depression is a neuropsychiatric ailment that has a major impact on socio-economic status. This disorder results from maladaptive neuroplastic processes in the cellular structures concerned with emotional processing, such as the prefrontal cortex (PFC), hippocampus and amygdala [18]. Neurotrophins and neuroplasticity are generally accepted to play an essential role in the pathophysiology of depression. However, the disturbance of synaptic plasticity due to glial cell pathology, particularly in the frontolimbic areas of the brain, is an emerging proposed mechanism that may substantially contribute to the pathophysiology of mood disorders [28].

Physical exercise can provide relief for depressed mood, but the underlying mechanisms remain uncertain. Studies designed to identify these mechanisms have discussed the changes in neurons with slight attention paid to changes in glial cells [11].

The term neuroglia describes all supporting non-neuronal cells in the central nervous system (CNS) that represent the homeostatic and defensive arm of nervous system [34]. Recently, a system of complex crosstalk between neurons and glia has been proposed, and the active role for glial cells in central homeostatic processes has been presented [29].

Astrocytes are a heterogeneous class of glial cells that preserve the neural microenvironment [7]. Their dynamic role in shaping synaptic function and their impact on behavioural expression are noteworthy [28]. Microglia are specific immune cells of the CNS that are highly dynamic, surveying the brain parenchyma and contributing to neuroprotection. During neurogenesis, microglia have a role in determining the ultimate patterns of the neural circuits important for behaviour and disease development [35]. These glia also have a critical role in the synaptic carving needed for adaptive brain function [37]. Oligodendroglia are myelin-forming cells that are generated continuously in healthy adult brains for regulation of the axonal function [20].

Experimental studies on the pathophysiology of depression usually focus on neuronal changes, with little attention has paid to changes in the neuroglia. This study aimed to consider changes in astrocytic counts and morphology in the hippocampus, dentate gyrus (DG), medial prefrontal cortex (MPFC) and cerebellar cortex of Wistar rats in an experimental depressive state and after voluntary running.

\section{MATERIALS AND METHODS}

This experimental study was conducted in the behaviour laboratory of the College of Medicine, Taibah University, Saudi Arabia. Cell counting and analysis were performed in the Department of Histology at the Bogomolets National Medical University, Ukraine. The animal handling was conducted in accordance with EC Directive 86/609/EEC for animal studies and the National Committee guidelines of Saudi Arabia.

\section{Experimental animals}

Fifty-five adult male Wistar rats aged 8-10 weeks and weighing 200-250 g were obtained from the animal house of the behaviour laboratory, housed in the laboratory for 7 days for acclimatisation, and observed for their movements and food consumption as markers of their ordinary behaviour. A 6-minute forced swimming test (FST) was performed for choosing rats with extreme test behaviour (less immobility duration and high distance moved) [1]. Selected animals ( $n=45$ rats) were housed in ventilated cages (5 animals/cage) with a standard chow diet and an adjusted laboratory environment (12/12-hour light/ /dark cycle, $22-24^{\circ} \mathrm{C}, 50-60 \%$ humidity) $[11,13]$.

Selected rats were randomly divided into three equal groups as follows:

- control group: rats were fed a standard chow diet for a period of 5 weeks;

- depression group: rats were subjected to the forced swimming programme for 2 weeks and then fed the standard chow diet for the following 3 weeks;

- depression/exercise group: rats were subjected to the forced swimming programme for 2 weeks and then allowed to voluntary wheel running for the following 3 weeks.

For all groups, the locomotor activities of the rats were assessed by the 6 -minute FST before being sacrificed.

\section{Induction of depression}

Depression was induced by forced swimming inside an upright glass cylinder inclosing water (water height $45 \mathrm{~cm}$ and temperature at $23-25^{\circ} \mathrm{C}$ ) for $15 \mathrm{~min}$ daily for 14 days. Every day after swimming, the rats were allowed to dry $\left(32^{\circ} \mathrm{C}\right)$ for $15 \mathrm{~min}$ before being returned to their cages [11].

\section{Accessing voluntary running}

The rats were allowed to run voluntarily for 3 weeks in rat running wheels supplied with counters 


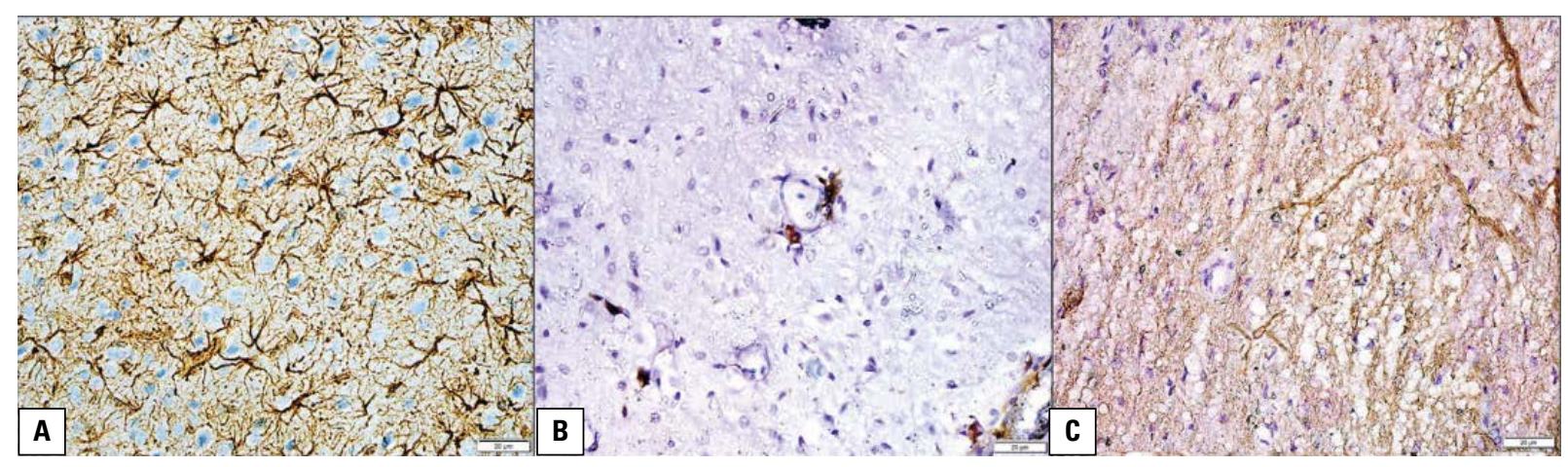

Figure 1. Photomicrographs of sections of the control medial frontal cortex stained for glial fibrillary acidic protein (GFAP) to detect astrocytes (A); myelin basic protein (MBP) to detect oligodendroglia (B); cluster of differentiation 68 (CD68) to detect microglia (C) (GFAP, MBP and CD68, $400 \times$ magnification).

(Lafayette Instrument Company, Inc. Lafayette, IN, USA).

\section{Confirmation of the development and/or the relief of the depressive state}

Two means were used to confirm the development or relief of a depressive state just before the rats were euthanised:

- the 6-minute FST, through automatic tracing via a software (Ethovision XT version 8.0, Noldus Information Technology, Wageningen, The Netherlands), was conducted to assess the locomotor behaviour of the rats in terms of a) immobility duration (in seconds) and b) the distance the rats moved (in centimetres) [26];

- quantification of serum corticosterone levels; in the morning, after anaesthetising the rats with ether, blood was collected from the retro-orbital veins and put into serum separation tubes for centrifugation. The sera were reserved in $-80^{\circ} \mathrm{C}$ until the levels of corticosterone (ALPCO Diagnostics, Orangeburg, NY, USA) were measured using ELISA kits rendering the manufacturers' instructions $[2,24]$.

\section{Tissue preparation and quantification of glial cells}

At the quantified time, the rats were sacrificed by cervical dislocation and perfused with formaldehyde (4\%) in phosphate-buffered saline (PBS). The brains were removed, coronally sectioned, and fixed in buffered formalin for histological examination of the hippocampus, DG, mPFC and cerebellar cortex. Brain tissue thin sections $(4-5 \mu \mathrm{m})$ were immunostained with polyclonal anti-rat antibodies (Dako Cytomation, USA) against glial fibrillary acidic protein (GFAP), myelin basic protein (MBP), and cluster of differentiation
68 (CD68). The staining procedure was performed as previously described [19] with an immunoperoxidase technique and a Bench-Mark instrument (Ventana Company, Oro Valley, Arizona, USA) briefly; The paraffin sections were deparaffinised, rehydrated, treated with $3 \% \mathrm{H}_{2} \mathrm{O}_{2}$ for $5 \mathrm{~min}$ and washed with PBS for $15 \mathrm{~min}$. The sections were blocked with $1.5 \%$ normal goat serum in PBS to control background staining and then incubated for $45 \mathrm{~min}$ at room temperature with the primary antibody. The sections were subsequently incubated with a second-stage biotinylated antibody at room temperature. After rinsing with PBS, the reaction products were visualised by immersing the sections in diamnobenzidine (DAB). Finally, the sections were counter-stained with haematoxylin, dehydrated and cover-slipped using Protex mounting media (DAB, Stock Stain box; Boster Biotechnology, Pleasanton, CA, USA) [16].

Sections were examined with a bright field automated microscope (Olympus BX 36 provided with a true-colour image analysis software package), photographs were captured from different brain sections to represent all strata of the hippocampus (alveus, oriens and radiatum and pyramidal), all layers of the DG (molecular, granular and polymorphic), all cell layers of the cerebellar cortex (molecular, Purkinje and granular) and all layers of the MPFC (molecular, outer granular, pyramidal, inner granular layer, ganglionic and multiform). The images were digitised by a DP27 digital video camera $(2448 \times 1920$-pixel matrix $)$ to identify different types of glial cells; GFAP + ve astroglia, MBP + ve oligodendroglia, and CD68 + ve microglia (Fig. 1) [19]. Cell counting was performed in the Department of Histology at the Bogomolets National Medical University, Ukraine. For each group, the stained astrocytes, oligodendroglia and microglia 

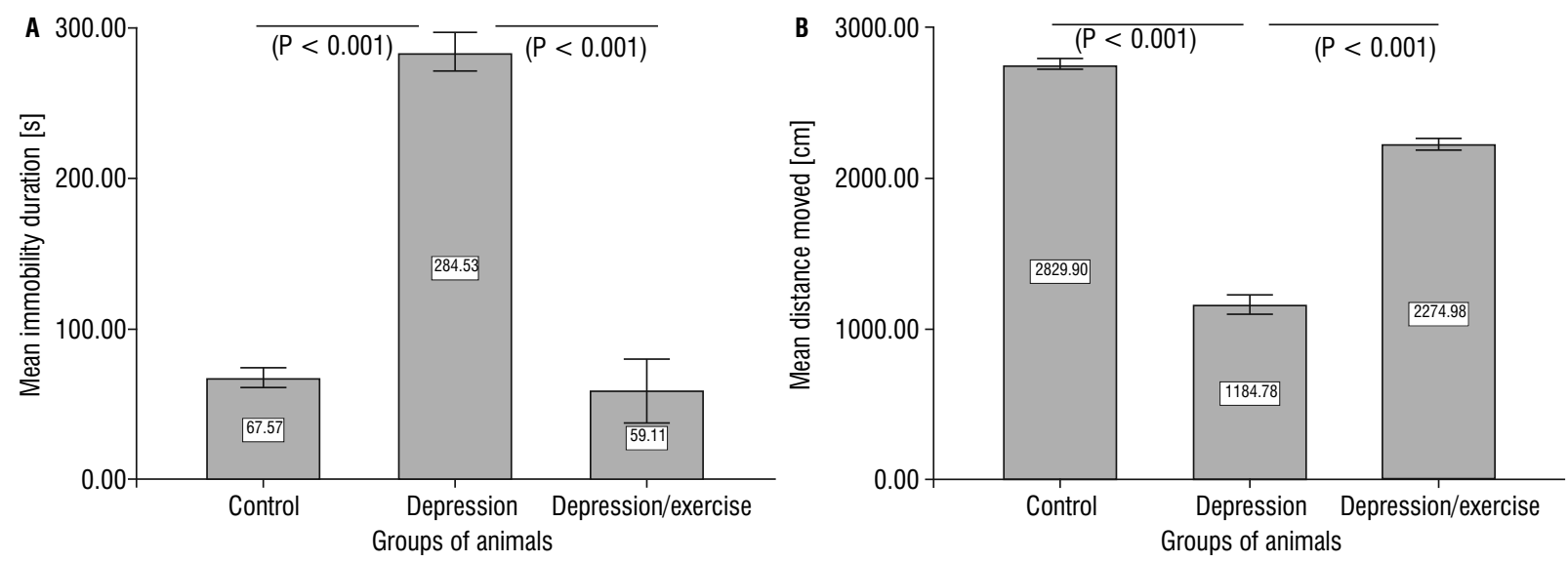

Figure 2. The results of behaviour tracings during the 6-minute forced swimming test for all groups: immobility duration (A) and distance moved (B).

were counted at $400 \times$ in 15 fields from the selected sections of the four brain regions.

\section{The glial astrocyte indices}

Three glial astrocyte indices (GAI) were calculated as a simple way to express the ratio of astrocytic count to the counts of other types of glia and to explore the impact of altered astrocytic numbers on glial structural homeostasis in the context of depression and also after voluntary running of depressed rats. They were expressed as follows: a) GAl1 (A/M) - number of astrocytes/the number microglia; b) GAI2 - number of astrocytes/number of oligodendroglia; and c) GAI3 (A/T) - number of astrocytes/total number of counted glial cells.

\section{The measurement of optical densities of the GFAP}

The optical densities (ODs) of the GFAP-stained sections were measured as described by Varghese et al. [32] in five illustrative non-overlapping fields $(\times 400)$. The images were deconvoluted via the DAB vector, the brown $D A B$ images were calibrated and the mean grey value. The OD was calculated using the equation: [OD $=\log$ (max intensity/mean intensity)], where max intensity $=250$ and mean intensity $=$ mean grey value.

\section{Statistical analysis}

Statistical analyses were done with the IBM SPSS statistical package (version 21). The data were stated as the mean \pm standard error (SE). The means of the studied groups were compared using one-way ANOVA with the least significant different test for analysis of the locomotor parameters, corticosterone levels, glial cell counts and glial astrocyte indices. Statistical significance was set at $p<0.05$.

\section{RESULTS}

The development and relief of the depressive state

The mean immobility duration recorded for the depression group was significantly higher than that for the control group. Further, the mean immobility duration recorded for the depression/exercise group was significantly lower than that for the depression group (Fig. 2).

The mean distance moved by the rats in the depression group was significantly lower than that moved by the rats in the control group. Also, the mean distance moved by the rats in the depression/ /exercise group was significantly higher than that recorded for the depression group (Fig. 2).

The mean serum corticosterone level was significantly higher in the depression group than in the control group. Furthermore, it was significantly lower in the depression/exercise group than in the depression group (Fig. 3).

\section{Glial cell counts and glial astrocyte indices}

The mean numbers and significance of the glial cells and glial astrocyte indices are presented in Table 1 and Table 2. Changes in the mean numbers of astrocytes in different regions for all groups are shown in Figure 4.

The mean number of astrocytes was significantly lower in the depression group than in the control group in all studied brain areas (hippocampus, DG, 


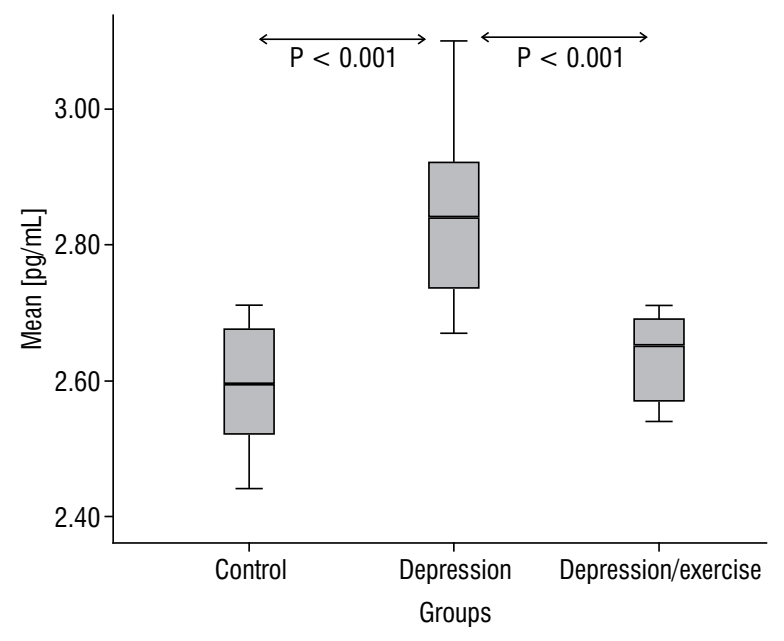

Figure 3. The changes in mean serum corticosterone levels in different groups.
mPFC and cerebellar cortex). However, no significant differences were detected for either oligodendroglia or microglia in the hippocampus, DG and MPFC between the depression group and the control group. Only oligodendroglia was significantly decreased in number in the cerebellar cortex.

Total glial cell counts were significantly lower in the depression group than in the control group in all studied brain areas except in the MPFC, where the decrease was not significant.

Excitingly, all glial astrocyte indices (GAI1, GAI2 and GAI3) were significantly lower in the depression groups than in the control group in all studied brain areas, except for a non-significant decrease in GAI1 and GAI3 in the cerebellar cortex.

Table 1. Means and significance of different glia cells' numbers and the total glia cells' counts in different groups in all examined brain areas

\begin{tabular}{|c|c|c|c|c|c|}
\hline Regions & Variables & Control (n = 15) & Depression (n = 15) & Depression/exercise $(n=15)$ & $P$ value \\
\hline \multirow[t]{4}{*}{ Dentate gyrus } & Astrocytes & $38.8 \pm 5.6$ & $11.8 \pm 3.1$ & $22.07 \pm 1$ & $\begin{array}{l}\mathrm{P} 1<0.001^{*} \\
\mathrm{P} 2=0.004^{*}\end{array}$ \\
\hline & Oligodendroglia & $11.47 \pm 2.1$ & $9.2 \pm 0.8$ & $98 \pm 10.5$ & $\begin{array}{l}\mathrm{P} 1=0.314 \\
\mathrm{P} 2<0.001^{*}\end{array}$ \\
\hline & Microgliocytes & $4.9 \pm 0.3$ & $5.7 \pm 0.7$ & $16 \pm 1.9$ & $\begin{array}{l}\mathrm{P} 1=0.255 \\
\mathrm{P} 2<0.001^{*}\end{array}$ \\
\hline & Total glia cells & $55.1 \pm 6.3$ & $26.7 \pm 3.2$ & $136.1 \pm 10.1$ & $\begin{array}{l}\mathrm{P} 1=0.007^{*} \\
\mathrm{P} 2<0.001^{*}\end{array}$ \\
\hline \multirow[t]{4}{*}{ Medial frontal cortex } & Astrocytes & $21 \pm 4$ & $9.2 \pm 3$ & $16.5 \pm 1.1$ & $\begin{array}{l}\mathrm{P} 1=0.027^{*} \\
\mathrm{P} 2=0.033^{*}\end{array}$ \\
\hline & Oligodendroglia & $7.7 \pm 1.2$ & $11.1 \pm 1.7$ & $30.5 \pm 5.6$ & $\begin{array}{c}\mathrm{P} 1=0.11 \\
\mathrm{P} 2=0.003^{*}\end{array}$ \\
\hline & Microgliocytes & $11.3 \pm 1.5$ & $8.9 \pm 0.8$ & $13.9 \pm 1.3$ & $\begin{array}{l}P 1=0.196 \\
P 2=0.004^{*}\end{array}$ \\
\hline & Total glia cells & $39.9 \pm 4.5$ & $29.2 \pm 3.7$ & $60.8 \pm 5.1$ & $\begin{array}{l}\mathrm{P} 1=0.098 \\
\mathrm{P} 2<0.001^{*}\end{array}$ \\
\hline \multirow[t]{4}{*}{ Hippocampus } & Astrocytes & $30.6 \pm 2.6$ & $15.7 \pm 2.8$ & $22.8 \pm 0.8$ & $\begin{array}{l}\mathrm{P} 1<0.001^{*} \\
\mathrm{P} 2=0.024^{*}\end{array}$ \\
\hline & Oligodendroglia & $21.7 \pm 2.6$ & $18.5 \pm 2.2$ & $26.7 \pm 0.8$ & $\begin{array}{l}P 1=0.368 \\
P 2=0.002^{*}\end{array}$ \\
\hline & Microgliocytes & $10.9 \pm 1.6$ & $10.7 \pm 1$ & $7.7 \pm 0.7$ & $\begin{array}{l}\mathrm{P} 1=0.918 \\
\mathrm{P} 2=0.018^{*}\end{array}$ \\
\hline & Total glia cells & $63.2 \pm 3.8$ & $45 \pm 4.1$ & $57.3 \pm 1.6$ & $\begin{array}{c}\mathrm{P} 1<0.001^{*} \\
\mathrm{P} 2=0.871\end{array}$ \\
\hline \multirow[t]{4}{*}{ Cerebellar cortex } & Astrocytes & $8 \pm 1.2$ & $4.33 \pm 0.4$ & $5.8 \pm 0.3$ & $\begin{array}{l}\mathrm{P} 1=0.007^{*} \\
\mathrm{P} 2=0.01^{*}\end{array}$ \\
\hline & Oligodendroglia & $238.3 \pm 14$ & $180 \pm 10.3$ & $353.3 \pm 15.2$ & $\begin{array}{l}\mathrm{P} 1=0.002^{*} \\
\mathrm{P} 2<0.001^{*}\end{array}$ \\
\hline & Microgliocytes & $4.2 \pm 0.4$ & $4.3 \pm 0.3$ & $4.7 \pm 0.3$ & $\begin{array}{l}P 1=0.777 \\
P 2=0.301\end{array}$ \\
\hline & Total glia cells & $250.5 \pm 14$ & $188 \pm 10.4$ & $363.8 \pm 15.2$ & $\begin{array}{l}\mathrm{P} 1=0.002^{*} \\
\mathrm{P} 2<0.001^{*}\end{array}$ \\
\hline
\end{tabular}

P1 significance of the depression group compared to the control group. Significance at $\mathrm{p}<0.05 ; \mathrm{P} 2$ significance of the depression/exercise group compared to the depression group. Significance at $p<0.05$. 
Table 2. Astrocytic indices and significance in different groups in all examined brain areas

\begin{tabular}{|c|c|c|c|c|c|}
\hline Regions & Glial astrocyte indices & Control $(n=15)$ & Depression $(n=15)$ & Depression/exercise $(n=15)$ & $P$ value \\
\hline \multirow[t]{3}{*}{ Dentate gyrus } & GAl1 (A/M) & $4.1 \pm 0.5$ & $1.6 \pm 0.5$ & $0.3 \pm 0.1$ & $\begin{array}{l}\mathrm{P} 1 \leq 0.001^{*} \\
\mathrm{P} 2=0.029^{*}\end{array}$ \\
\hline & GAI2 (A/0) & $8.4 \pm 1.3$ & $2.3 \pm 0.7$ & $1.6 \pm 0.1$ & $\begin{array}{l}\mathrm{P} 1<0.001^{*} \\
\mathrm{P} 2=0.534\end{array}$ \\
\hline & GAl3 (A/Total) & $0.7 \pm 0.04$ & $0.4 \pm 0.1$ & $0.2 \pm 0.03$ & $\begin{array}{l}\mathrm{P} 1<0.001^{*} \\
\mathrm{P} 2=0.022^{*}\end{array}$ \\
\hline \multirow[t]{3}{*}{ Medial frontal cortex } & GAl1 (A/M) & $3.8 \pm 0.8$ & $1 \pm 0.3$ & $0.8 \pm 0.1$ & $\begin{array}{c}\mathrm{P} 1<0.001^{*} \\
\mathrm{P} 2=0.805\end{array}$ \\
\hline & GAI2 (A/0) & $2.7 \pm 0.8$ & $1 \pm 0.4$ & $1.4 \pm 0.2$ & $\begin{array}{c}\mathrm{P} 1=0.023^{*} \\
\mathrm{P} 2=0.640\end{array}$ \\
\hline & GAl3 (A/Total) & $0.5 \pm 0.1$ & $0.2 \pm 0.1$ & $0.3 \pm 0.02$ & $\begin{array}{c}\mathrm{P} 1<0.001^{*} \\
\mathrm{P} 2=0.269\end{array}$ \\
\hline \multirow[t]{3}{*}{ Hippocampus } & GAl1 (A/M) & $2.6 \pm 0.8$ & $0.9 \pm 0.2$ & $0.7 \pm 0.1$ & $\begin{array}{c}P 1=0.015^{*} \\
P 2=0.872\end{array}$ \\
\hline & GAI2 (A/0) & $7.5 \pm 1.6$ & $2.7 \pm 0.5$ & $2.5 \pm 0.2$ & $\begin{array}{l}P 1=0.001^{*} \\
P 2=0.899\end{array}$ \\
\hline & GAl3 (A/Total) & $0.5 \pm 0.3$ & $0.3 \pm 0.05$ & $0.4 \pm 0.01$ & $\begin{array}{l}\mathrm{P} 1<0.001^{*} \\
\mathrm{P} 2=0.052\end{array}$ \\
\hline \multirow[t]{3}{*}{ Cerebellar cortex } & GAl1 (A/M) & $0.04 \pm 0.007$ & $0.03 \pm 0.003$ & $0.02 \pm 0.002$ & $\begin{array}{l}P 1=0.072 \\
P 2=0.165\end{array}$ \\
\hline & GAI2 (A/0) & $1.9 \pm 0.3$ & $1.02 \pm 0.1$ & $1.3 \pm 0.1$ & $\begin{array}{c}\mathrm{P} 1<0.001^{*} \\
\mathrm{P} 2=0.291\end{array}$ \\
\hline & GAI3 (A/Total) & $0.03 \pm 0.006$ & $0.02 \pm 0.003$ & $0.02 \pm 0.002$ & $\begin{array}{l}P 1=0.061 \\
P 2=0.192\end{array}$ \\
\hline
\end{tabular}

GAl1 (A/M) - number of astrocytes/number of microglia; GAI2 (A/0) — number of astrocytes/number of oligodendroglia; GAl3 $(\mathrm{A} / \mathrm{T})$ - number of astrocytes/total number of counted glial cells; P1 significance of the depression group compared to the control group; P2 significance of the depression/exercise group compared to the depression group. Significance at $\mathrm{p}<0.05$.

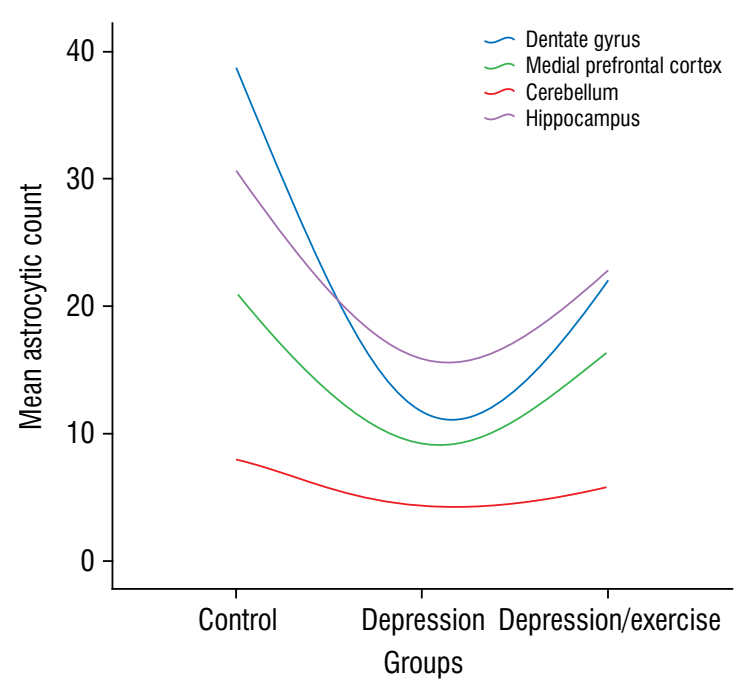

Figure 4. The changes in mean astrocytic counts in different brain areas.

The mean numbers of astrocytes and oligodendroglia were significantly higher in the depression/ /exercise group than in the depression group in all studied brain areas (hippocampus, DG, mPFC and cerebellar cortex). The mean number of microglia was different in different regions (increased significantly in the DG and MPFC, decreased significantly in the hippocampus, and no significant increase in the cerebellar cortex).

Total glial cell counts were significantly higher in the depression/exercise group than in the depression group in all studied brain areas except in the hippocampus, where the increase was not significant.

However, the glial astrocyte indices showed no significant differences in the hippocampus, mPFC or cerebellar cortex between the depression and depression exercise groups. In the DG, significant decreases in GAl1 and GAl3 were detected, but these decreases were accompanied by highly significant increases in the numbers of microglia and oligodendroglia.

\section{GFAP immunohistochemical study}

An examination of the hippocampus, DG, mPFC and cerebellar cortex in the control group revealed star-shaped astrocytes with dense, distinct, dark cell bodies and complex and "bushy", densely packed processes (Fig. 5). In depressed rats, astrocytes were 


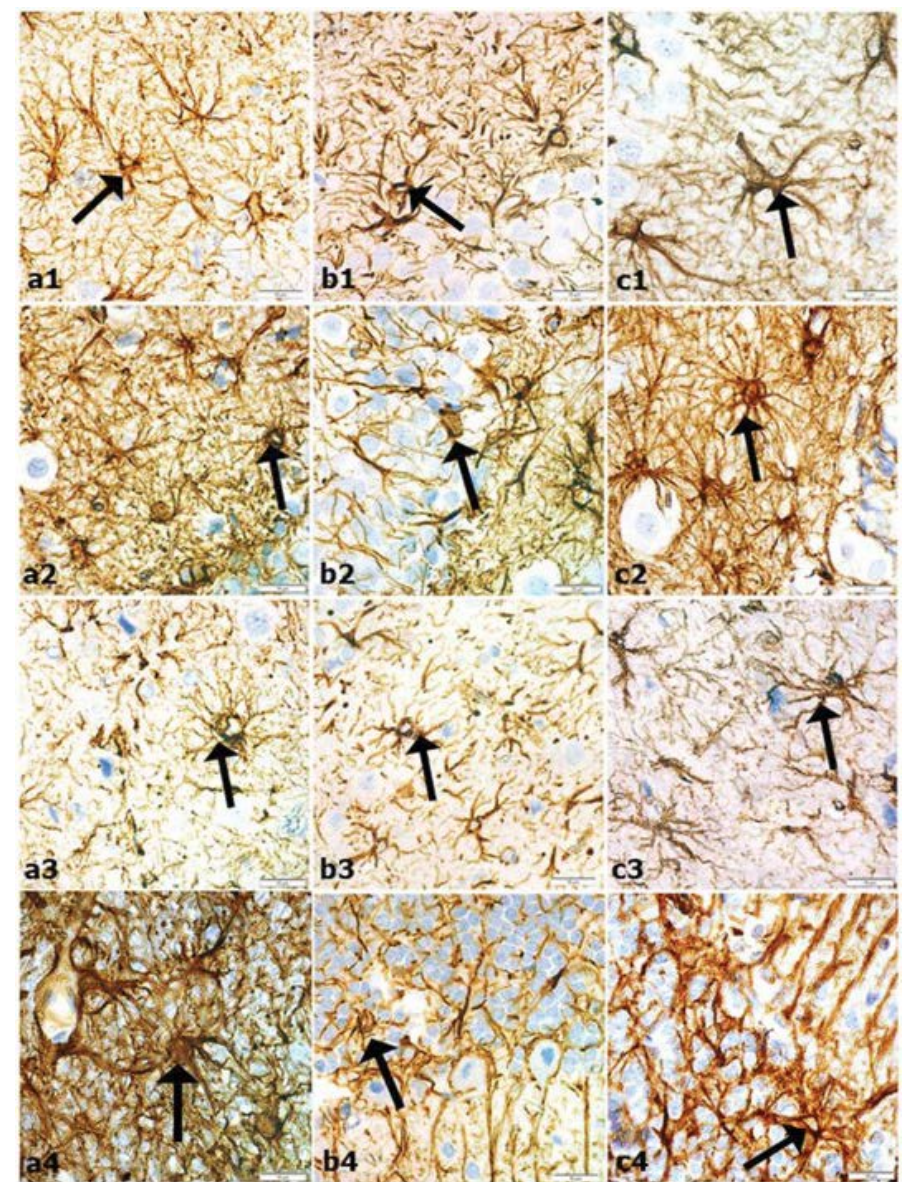

Figure 5. Plate showing immunohistochemistry for glial fibrillary acidic protein (GFAP)-positive cells (arrows) in the brain areas of different groups. In the control group (a), astrocytes display dense, distinct, dark cell bodies and "bushy", densely packed processes in the hippocampus (a1), dentate gyrus (DG; a2), medial prefrontal cortex (mPFC; a3) and cerebellar cortex (a4). In the depression group (b), the astrocytes appear dispersed and display relatively indistinct cell bodies and distorted processes in the hippocampus (b1), DG (b2), mPFC (b3) and cerebellar cortex (b4). In the depression/exercise group (c), the astrocytes appeared to regain their immunoreactivity, exhibiting dense cell bodies and complex, dense process extensions in the hippocampus (c1), DG (c2), mPFC (c3) and cerebellar cortex (c4) (GFAP, $1000 \times$ magnification).

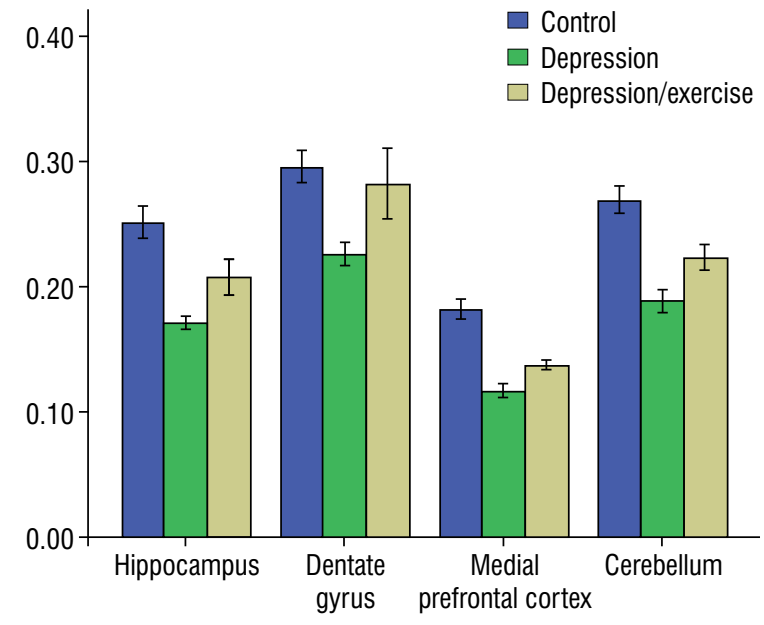

Figure 6. The mean optical density values of glial fibrillary acidic protein immunostaining in different brain areas of different groups.

dispersed in all examined brain regions and displayed relatively indistinct cell bodies and distorted processes (Fig. 5). In the depression/exercise group, astrocytes in all regions appeared to regain their immune reactivity, exhibiting denser cell bodies and complex, denser process extensions than the depression group (Fig. 5).
Compared to the control group, depression caused a significant decrease in GFAP OD in the hippocampus $(p=0.013)$, DG $(p<0.001), \operatorname{mPFC}(p<0.001)$, and cerebellar cortex $(p<0.001)$ (Fig. 6).

Compared to the depression group, running caused a significant increase in GFAP OD in the hippocampus $(p=0.042)$, DG $(p=0.027)$, mPFC $(p=0.037)$, and cerebellar cortex $(p=0.026)$ (Fig. 6).

\section{DISCUSSION}

The development of depression and/or its relief were assessed both mechanically by evaluating locomotor behaviours (through FST) or biochemically by evaluating the serum corticosterone levels in all groups. The significant decrease of the distance moved, the significant increase in the immobility duration and the increased corticosterone levels after the forced swimming protocol confirmed the development of depression. Similarly, a significant increase in the mean distance moved, a significant decrease in the mean immobility duration and a significant decrease in serum cortisone levels occurred after a voluntary running protocol confirmed the relief of depressive behaviour $[3,5]$. 
Depression was primarily proposed to develop due to increased levels of corticosteroids [21]; later, neurotrophic factors and disordered neuroplastic processes in the frontal cortex and limbic system were proposed as underlying mechanisms $[6,11]$. Based upon the emerging concept that glial cells, specifically astrocytes, are critical factors affecting brain plasticity [28], this study evaluated the changes in glial cells (specifically astrocytes) in the hippocampus, DG, mPFC, and cerebellar cortex as a key mechanism triggering the pathophysiology of depression.

The significant decrease in the mean total glial cell counts in the hippocampus, DG, and cerebellar cortex reflected the contribution of glial cells to the pathophysiology of depression. This finding was consistent with a recorded reduction in glia during depression that was proven to precede the decreased neuronal density, which appears to occur later after longer periods of depression [23].

The significant decrease in the mean number of astrocytes, along with non-significant differences in the mean count of oligodendroglia or microglia, in most of the examined brain areas, can reflect the major role of astrocytes in the pathology of depression. Additionally, the decreased glial astrocyte indices can outline the role of astrocytes over other glia in the development of depression. These findings are in consistent with the reported decreased density of astrocytes associated with synaptic dysfunction in the frontolimbic regions during depression [28] and in the hippocampus during chronic stress [2].

It is imperative to consider that the contribution of glial cells to the pathogenesis of depression is not contradicted by the old theories of depression that attributed the development of depression to the raised corticosterone or deficits in neurotrophic factors $[6,18]$ as these factors have an impact on glial function [28]; however, the role of astrocytes and glial-glial interactions in controlling depressive behaviour constitutes a new era of neuroscience research. These cells play major roles in synaptic function in specified brain regions $[9,25]$, and have tough interactions with the synapses that make it difficult to distinguish whether the dysfunction resulted from neuronal or astrocyte modifications [33].

In accordance with our results, some evidence supports the idea that astrocyte modification in some brain areas leads to depression [27]. In humans, post-mortem brain samples from adults suffering from major depressive disorder showed a decreased number of astrocytes in the frontolimbic structures [31].
The significant decrease in the OD of GFAP immune-expression in the brain areas in the depression group was consistent with other studies that described a modification in the density of different astrocytic markers in the frontolimbic regions in major depressive disorder [14]. Moreover, GFAP-expressing cells are decreased in the PFC after 5 weeks of chronic stress [2] and in genetically stressed Wistar Kyoto rats [15]. Furthermore, postmortem brain samples showed decreased GFAP expression in the hippocampus and PFC, with no neuronal effects, although defective neuronal metabolism or connections have been suggested [36].

Regarding the cerebellum, the significant reduction in the number of astrocytes and in the OD of GFAP in the cerebellar cortex of rats in the depression group could represent an indication for the involvement of the cerebellar cortex in the pathophysiology of depression, as previously suggested [12]. One possible explanation is the known anatomical and functional connections of the cerebellum to the PFC and the subcortical limbic structures [17]. Nonetheless, in the cerebellum, oligodendroglia are involved in cerebellar pathology, with a significant decrease in their mean count in the depression group, which could explain the non-significant decreases in GAl1 and GAI3 that were specifically observed in the cerebellar cortex.

Practising voluntary running by depressed rats improved the depressive locomotor behaviour and produced a significant increase in the total glial cell counts and significant increase of the OD of the GFAP-stained sections in the brain areas. This can indicate the concomitant improving effect on glial cell counts with the improvement of the depressive behaviour after voluntary running. This was consistent with the observed alleviation of depression-like symptoms and the enhancement of synaptic, and glial measures in several brain regions upon exercise [34].

The increased number of astrocytes in the MPFC, hippocampus and DG, along with improved depressive behaviour after voluntary running, are in line with the suggested physical activity-induced plasticity of astrocytes and enhanced synaptic markers in the brain regions for cognition [30]. The improved behaviour might be due to the trophic support and regulation of neuronal blood flow by astrocytes, which influence neuronal function and synaptic growth [12, 22].

Also, the improved astrocytic counts and increased GFAP expression after running are consistent with the suggested important glioprotective effects and 
the prevention of stress-induced decreases in GFAP expression in the hippocampus after treatment with antidepressants [8].

Notably, although astrocyte counts increased in all brain areas after running, there were non-significant increases in the proposed glial astrocyte indices in the hippocampus, mPFC and cerebellar cortex. This finding showed that voluntary running increased the astrocyte count to levels capable of preserving neurons and oligodendroglia, limiting brain tissue degeneration and preserving the function without reaching the levels of astrogliosis, which can lead to neurotoxicity, potential neural dysfunction and the development of neurodegeneration [4]. The significant decreases in GAI1 and GAI3 in the DG can be explained by the specifically significant increase in microglia to this region, which might be enhanced to help shaping the final patterns of neural connections during neurogenesis $[10,35]$.

\section{CONCLUSION}

These collected data provide evidence for a prominent role of glial cells in the pathology of depression. Furthermore, the study presents significant changes in astrocyte count and/or morphology in the hippocampus, DG, mPFC and cerebellar cortex in depression. The study reveals the noteworthy ability of voluntary running to reverse the effects of depression on the abundance of astrocytes and on their specific marker GFAP in the brain regions associated with depression. Additionally, this work provides evidence that both astrocytes and oligodendroglia are implicated in the mitigating effect of voluntary running on depression in the hippocampus, DG, mPFC and cerebellar cortex.

\section{Acknowledgement}

The authors express thanks for the technicians of the behavioural lab, College of Medicine, Taibah University, who helped us throughout conduction of the experiment.

\section{REFERENCES}

1. Andrus BM, Blizinsky K, Vedell PT, et al. Gene expression patterns in the hippocampus and amygdala of endogenous depression and chronic stress models. Mol Psychiatry. 2012; 17(1): 49-61, doi: 10.1038/mp.2010.119, indexed in Pubmed: 21079605.

2. Araya-Callís C, Hiemke C, Abumaria N, et al. Chronic psychosocial stress and citalopram modulate the expression of the glial proteins GFAP and NDRG2 in the hippocampus. Psychopharmacology (Berl). 2012; 224(1):
209-222, doi: 10.1007/s00213-012-2741-x, indexed in Pubmed: 22610521.

3. Ayuob N, Ali S, Suliaman M, et al. The antidepressant effect of musk in an animal model of depression: a histopathological study. Cell Tissue Res. 2016; 366(2): 271-284, doi: 10.1007/s00441-016-2468-9.

4. Barres BA. The mystery and magic of glia: a perspective on their roles in health and disease. Neuron. 2008; 60(3): 430-440, doi: 10.1016/j.neuron.2008.10.013, indexed in Pubmed: 18995817.

5. Bernardi C, Tramontina AC, Nardin P, et al. Treadmill exercise induces hippocampal astroglial alterations in rats. Neural Plast. 2013; 2013: 709732, doi: 10.1155/2013/709732, indexed in Pubmed: 23401802.

6. Castrén E. Neurotrophins and psychiatric disorders. Handb Exp Pharmacol. 2014; 220: 461-479, doi: 10.1007/978-3642-45106-5_17, indexed in Pubmed: 24668483.

7. Constantinescu CS, Tani M, Ransohoff RM, et al. Astrocytes as antigen-presenting cells: expression of IL-12/IL-23. J Neurochem. 2005; 95(2): 331-340, doi: 10.1111/j.14714159.2005.03368.x, indexed in Pubmed: 16086689.

8. Czéh B, Simon M, Schmelting B, et al. Astroglial plasticity in the hippocampus is affected by chronic psychosocial stress and concomitant fluoxetine treatment. Neuropsychopharmacology. 2006; 31(8): 1616-1626, doi: 10.1038/ sj.npp.1300982, indexed in Pubmed: 16395301.

9. Duman RS, Aghajanian GK. Synaptic dysfunction in depression: potential therapeutic targets. Science. 2012; 338(6103): 68-72, doi: 10.1126/science.1222939, indexed in Pubmed: 23042884.

10. Ehninger D, Kempermann G. Regional effects of wheel running and environmental enrichment on cell genesis and microglia proliferation in the adult murine neocortex. Cereb Cortex. 2003; 13(8): 845-851, doi: 10.1093/ cercor/13.8.845, indexed in Pubmed: 12853371.

11. Eldomiaty MA, Almasry SM, Desouky MK, et al. Voluntary running improves depressive behaviours and the structure of the hippocampus in rats: A possible impact of myokines. Brain Res. 2017; 1657: 29-42, doi: 10.1016/j. brainres.2016.12.001, indexed in Pubmed: 27919728.

12. Fatemi SH, Laurence JA, Araghi-Niknam M, et al. Glial fibrillary acidic protein is reduced in cerebellum of subjects with major depression, but not schizophrenia. Schizophr Res. 2004; 69(2-3): 317-323, doi: 10.1016/j. schres.2003.08.014, indexed in Pubmed: 15469203.

13. Garrett $L$, Lie DC, Hrabé de Angelis $M$, et al. Voluntary wheel running in mice increases the rate of neurogenesis without affecting anxiety-related behaviour in single tests. BMC Neurosci. 2012; 13: 61, doi: 10.1186/1471-2202-13-61, indexed in Pubmed: 22682077.

14. Gittins RA, Harrison PJ. A morphometric study of glia and neurons in the anterior cingulate cortex in mood disorder. J Affect Disord. 2011; 133(1-2): 328-332, doi: 10.1016/j. jad.2011.03.042, indexed in Pubmed: 21497910.

15. Gosselin RD, Gibney S, O'Malley D, et al. Region specific decrease in glial fibrillary acidic protein immunoreactivity in the brain of a rat model of depression. Neuroscience. 2009; 159(2): 915-925, doi: 10.1016/j.neuroscience.2008.10.018, indexed in Pubmed: 19000745.

16. Grizzle WE. Special symposium: fixation and tissue processing models. Biotech Histochem. 2009; 84(5): 
185-193, doi: 10.3109/10520290903039052, indexed in Pubmed: 19886755.

17. Konarski JZ, McIntyre RS, Grupp LA, et al. Is the cerebellum relevant in the circuitry of neuropsychiatric disorders? J Psychiatry Neurosci. 2005; 30(3): 178-186, indexed in Pubmed: 15944742

18. Krishnan V, Nestler EJ. The molecular neurobiology of depression. Nature. 2008; 455(7215): 894-902, doi: 10.1038/ nature07455, indexed in Pubmed: 18923511.

19. Lyck L, Dalmau I, Chemnitz J, et al. Immunohistochemical markers for quantitative studies of neurons and glia in human neocortex. J Histochem Cytochem. 2008; 56(3): 201-221, doi: 10.1369/jhc.7A7187.2007, indexed in Pubmed: 17998570.

20. McKenzie IA, Ohayon D, Li H, et al. Motor skill learning requires active central myelination. Science. 2014; 346(6207): 318-322, doi: 10.1126/science.1254960, indexed in Pubmed: 25324381.

21. Meyer RM, Burgos-Robles A, Liu E, et al. A ghrelin-growth hormone axis drives stress-induced vulnerability to enhanced fear. Mol Psychiatry. 2014; 19(12): 1284-1294, doi: $10.1038 / \mathrm{mp} .2013 .135$, indexed in Pubmed: 24126924

22. Miguel-Hidalgo JJ, Baucom C, Dilley G, et al. Glial fibrillary acidic protein immunoreactivity in the prefrontal cortex distinguishes younger from older adults in major depressive disorder. Biol Psychiatry. 2000; 48(8): 861-873, doi: 10.1016/s0006-3223(00)00999-9, indexed in Pubmed: 11063981.

23. Miguel-Hidalgo JJ, Whittom A, Villarreal A, et al. Apoptosis-related proteins and proliferation markers in the orbitofrontal cortex in major depressive disorder. J Affect Disord. 2014; 158: 62-70, doi: 10.1016/j.jad.2014.02.010, indexed in Pubmed: 24655767.

24. Pariante CM. Depression, stress and the adrenal axis. J Neuroendocrinol. 2003; 15(8): 811-812, doi: 10.1046/j.13652826.2003.01058.x, indexed in Pubmed: 12834443.

25. Pekny M, Pekna M, Messing A, et al. Astrocytes: a central element in neurological diseases. Acta Neuropathol. 2016; 131(3): 323-345, doi: 10.1007/s00401-015-1513-1, indexed in Pubmed: 26671410.

26. Porsolt RD, Brossard G, Hautbois C, et al. Rodent models of depression: forced swimming and tail suspension behavioral despair tests in rats and mice. Curr Protoc Neurosci. 2001; Chapter 8: Unit 8.10A, doi: 10.1002/0471142301. ns0810as14, indexed in Pubmed: 18428536.

27. Rajkowska G, Stockmeier CA. Astrocyte pathology in major depressive disorder: insights from human postmortem brain tissue. Curr Drug Targets. 2013; 14(11): 1225-1236, doi: 10.2174/13894501113149990156, indexed in Pubmed: 23469922

28. Rial $D$, Lemos $C$, Pinheiro $H$, et al. Depression as a glial-based synaptic dysfunction. Front Cell Neurosci. 2015; 9: 521, doi: 10.3389/fncel.2015.00521, indexed in Pubmed: 26834566 .

29. Schafer DP, Lehrman EK, Stevens B. The "quad-partite" synapse: microglia-synapse interactions in the developing and mature CNS. Glia. 2013; 61(1): 24-36, doi: 10.1002/ glia.22389, indexed in Pubmed: 22829357.

30. Stranahan AM, Khalil D, Gould E. Running induces widespread structural alterations in the hippocampus and entorhinal cortex. Hippocampus. 2007; 17(11): 1017-1022, doi: 10.1002/hipo.20348, indexed in Pubmed: 17636549.

31. Torres-Platas SG, Nagy C, Wakid M, et al. Glial fibrillary acidic protein is differentially expressed across cortical and subcortical regions in healthy brains and downregulated in the thalamus and caudate nucleus of depressed suicides. Mol Psychiatry. 2016; 21(4): 509-515, doi: 10.1038/ mp.2015.65, indexed in Pubmed: 26033239.

32. Varghese $F$, Bukhari $A B$, Malhotra $R$, et al. IHC Profiler: an open source plugin for the quantitative evaluation and automated scoring of immunohistochemistry images of human tissue samples. PLoS One. 2014; 9(5): e96801, doi: 10.1371/journal.pone.0096801, indexed in Pubmed: 24802416.

33. Verkhratsky A, Steardo L, Parpura V, et al. Translational potential of astrocytes in brain disorders. Prog Neurobiol. 2016; 144: 188-205, doi: 10.1016/j.pneurobio.2015.09.003, indexed in Pubmed: 26386136.

34. Verkhratsky A, Zorec R, Parpura V. Stratification of astrocytes in healthy and diseased brain. Brain Pathol. 2017; 27(5): 629-644, doi: 10.1111/bpa.12537, indexed in Pubmed: 28805002.

35. Wake H, Moorhouse AJ, Nabekura J. Functions of microglia in the central nervous system--beyond the immune response. Neuron Glia Biol. 2011; 7(1): 47-53, doi: 10.1017/ S1740925X12000063, indexed in Pubmed: 22613055.

36. Webster MJ, Knable MB, Johnston-Wilson N, et al. Immunohistochemical localization of phosphorylated glial fibrillary acidic protein in the prefrontal cortex and hippocampus from patients with schizophrenia, bipolar disorder, and depression. Brain Behav Immun. 2001; 15(4): 388-400, doi: 10.1006/ brbi.2001.0646, indexed in Pubmed: 11782105.

37. Zhan Y, Paolicelli RC, Sforazzini F, et al. Deficient neuron-microglia signaling results in impaired functional brain connectivity and social behavior. Nat Neurosci. 2014; 17(3): 400-406, doi: 10.1038/nn.3641, indexed in Pubmed: 24487234. 